\title{
Distributed Differential Quasi-Orthogonal Space-Time Block Coded System for Multiple-Relay Networks
}

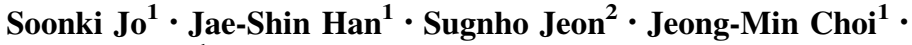 \\ Jong-Soo Seo ${ }^{1}$
}

(C) The Author(s) 2015. This article is published with open access at Springerlink.com

\begin{abstract}
In this paper, we present an efficient distributed differential quasi-orthogonal space-time block code (DD-QOSTBC) system for multiple relay networks. First, we propose the DD-QOSTBC transmission which considers two robust STBC-like subsystems in amplify-and-forward multiple relaying over flat fading channel. It is assumed that source has two antennas, and relays and destination have a single antenna. With robust STBC-like subsystem structure, we show that our robust subsystems can be used for an efficient joint suboptimal differential decoding based on a maximum likelihood criterion. Finally, we accomplish the performance evaluation on the proposed DD-QOSTBC system in terms of bit-error-rate.
\end{abstract}

Keywords Amplify-and-forward · Differential space-time block code · Quasi-orthogonal space-time block code

Jae-Shin Han

hjs4015@yonsei.ac.kr

Soonki Jo

joangel21@yonsei.ac.kr

Sugnho Jeon

jeonsh@kbs.co.kr

Jeong-Min Choi

choi_jm@yonsei.ac.kr

Jong-Soo Seo

jsseo@yonsei.ac.kr

1 Department of Electrical and Electronic Engineering, Yonsei University, Seoul 120-749, Korea

2 Technical Research Institute, Korean Broadcasting System (KBS), Seoul 150-179, Korea 


\section{Introduction}

Differential modulation has been regarded as an attractive solution to improve the spectral efficiency with pilot signal elimination at the transmitter. The potential goal of differential modulation is achieved in fast-varying fading channels. It is difficult to obtain the channel state information (CSI) between transceivers in those channels but differential modulation can be adopted without channel estimations. Due to this property, differential modulation is used for timing and frequency synchronization in order to initiate the frame decoding [1].

Recently, multiple-input multiple-output (MIMO) wireless systems have been developed to increase total reliability and spectral efficiency [2]. In particular, the advantages of MIMO systems, such as capacity and diversity improvements are compared with singleinput single-output (SISO) system adopting the combination of the differential modulation and an orthogonal space time block code (STBC) [3-5]. Unfortunately, however, employing a large number of antennas has been considered as an impractical direction for small mobile terminals due to its limited physical hardware size. Thus, the distributed diversity (or relay-assisted diversity) topology has aroused great interests for an alternative solution to overcome such shortcomings [6,7]. The diversity gain is organizationally obtained by joint processing using received signals from the distributed relays and transmitter. However, we note that most of prior works for cooperative system have been built upon perfect CSI transmission (coherent transmission). Furthermore, to the best of our knowledge, distributed differential modulation with quasi-orthogonal space-time block coded (D-QOSTBC) system has not achieved full diversity.

In this paper, we present a distributed D-QOSTBC (DD-QOSTBC) system with an amplify-and-forward (AF) mode for multiple half-duplex relaying (HDR) system. Especially, we consider a single frequency relaying system shown in Fig. 1a which assumes that the source $(S)$ has two antennas and each of $N$ relays $(R)$ has one antenna and the destination $(D)$ has a single antenna. Here, we firstly propose a novel robust STBC-like subsystems which preserve orthogonality on both effective channel and symbol matrices to reduce receiver complexity without performance degradations. With the robust subsystems, we propose the efficient joint suboptimal differential decoding (JSDD) utilizing a maximum likelihood (ML) criterion. This JSDD rapidly updates both error distances of the detected DD-QOSTBC symbols and additional information to calculate weighting factor. The weighting factor is combined with additional signals efficiently. Finally, we evaluate the performance of the proposed DD-QOSTBC system to compare with that of the distributed coherent QOSTBC (DC-QOSTBC) system, and verify the system's validity.

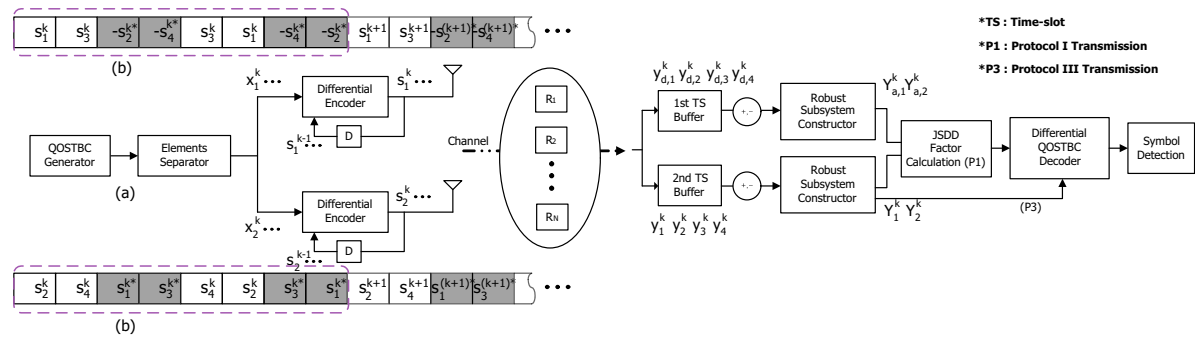

Fig. 1 DD-QOSTBC system consisted of source $(S)$, relays $(R)$, and destination $(D)$; a transceiver topology, b DD-QOSTBC transmission format 


\section{A Signal Model for Efficient DD-QOSTBC System}

In this section, we address a fundamental framework of multiple HDR system to realize the efficient differential encoding and decoding for the DD-QOSTBC system. First, we briefly describe Protocol I and III to achieve STBC diversity [7]: In Protocol I, the source communicates with the relays and destination during Time-slot 1. And the relays and source communicate with the destination during Time-slot 2. Protocol III has similar process with Protocol I but $S \rightarrow D$ link during Time-slot 1 is not considered. In the followings, Fig. 1a shows that the overall block diagram of DD-QOSTBC for multiple relay networks consists of one $S, N$ distributed HDRs, and single $D$. The modulated data passes QOSTBC generator and four symbols of the $k$ th block time $s_{1}^{k}, s_{2}^{k}, s_{3}^{k}$, and $s_{4}^{k}$ construct an QOSTBC matrix pattern as shown in (8). Then, $4 \times 4$ QOSTBC is divided into two blocks and differential modulation is performed with elements separator for symbol transmission. The constructed transmission blocks from each antenna are illustrated in Fig. 1b. It is noted that we omit the process of symbol-based differential modulation in this section because Sect. 4 will discuss the differential process at the destination side. As in Fig. 1b, differentially modulated symbols for the $k$ th block time in each antennas consists of eight symbols that 16 time slots are required to transmit QOSTBC codes successfully. Throughout this paper, we assume that all links have a Rayleigh distribution of $\mathcal{C N} \sim\left(0, \sigma_{h}^{2}\right)$.

\subsection{Transmission Procedures with Protocol III}

(1) During Time-slot 1 As shown in Fig. 1a, the $S$ transmits the symbols $s_{1}^{k}$ and $s_{2}^{k}$ to the $n$th relay. The received signal $r_{n}^{k}$ from two antennas of $S$ can be expressed as

$$
r_{n}^{k}=\sqrt{E_{s r}} h_{s r, 1 n}^{k} s_{1}^{k}+\sqrt{E_{s r}} h_{s r, 2 n}^{k} s_{2}^{k}+n_{R, n}^{k},
$$

where $E_{s r}$ denotes the average power available at $R$ and $h_{s r, i n}^{k}$ denotes a channel gain between the $i$ th transmit antenna of $S$ and the $n$th $R$. It is assumed that the additive noise of $n$th $R, n_{R, n}^{k}$ has the Gaussian distribution of $\mathcal{C N} \sim\left(0, \sigma_{n}^{2}\right)$ where $E\left[n_{R, n}^{k} n_{R, n}^{k \dagger}\right]=\sigma_{n}^{2}$. $(\cdot)^{\dagger}$ denotes hermitian operation. The received signal of (1) is normalized at each relay for the AF relaying transmission as

$$
\tilde{r}_{n}^{k}=\frac{1}{\eta}\left(\sqrt{E_{s r}} h_{s r, 1 n}^{k} s_{1}^{k}+\sqrt{E_{s r}} h_{s r, 2 n}^{k} s_{2}^{k}+n_{R, n}^{k}\right),
$$

where the factor $\eta=\sqrt{2 E_{s r} \sigma_{s}^{2}+\sigma_{n}^{2}}$ is a unit power at the $n$th $R$, and $\sigma_{s}^{2}$ is an approximated symbol variance for differential transmission [8].

(2) During Time-slot $2 S$ transmits symbols $s_{3}^{k}$ and $s_{4}^{k}$ to $D$ while each relays forwards the signal (2) to $D$. As a result, the received signal from both $S$ and $R$ can be expressed as

$$
y_{1}^{k}=\sum_{n=1}^{N} \tilde{r}_{n}^{k} h_{r d, n}^{k}+\sqrt{E_{s d}}\left(h_{s d, 1}^{k} s_{3}^{k}+h_{s d, 2}^{k} s_{4}^{k}\right)+n_{D 2}^{k},
$$

where $N$ is the number of distributed relays, and $h_{r d, n}^{k}$ denotes a channel gain between the $n$th $R$ and $D . n_{D 2}^{k}$ is an additive white Gaussian noise (AWGN) with $\mathcal{C N} \sim\left(0, \sigma_{n}^{2}\right)$. (3) can be rewritten as

$$
y_{1}^{k}=\zeta_{1}^{k} s_{1}^{k}+\zeta_{2}^{k} s_{2}^{k}+\zeta_{3}^{k} s_{3}^{k}+\zeta_{4}^{k} s_{4}^{k}+n_{1}^{k}
$$


where the effective channels $\zeta_{i}^{k}$ (for $\left.i=1,2,3,4\right)$ are specifically defined in (5), and the effective noise $n_{1}^{k}$ is specified as $n_{i}^{k}=\sqrt{E_{r d}} / \eta\left(\sum_{n=1}^{N} n_{R, 1}^{(k+i-1)} h_{r d, n}^{k}\right)+n_{D 2}^{(k+i-1)}$ (for $i=1$ ).

$$
\mathbf{h}^{k}=[\underbrace{\frac{\sqrt{E_{s r} E_{r d}}}{\eta} \sum_{n=1}^{N}\left(h_{s r, 1 n}^{k} h_{r d, n}^{k}\right)}_{\zeta_{1}^{k}} \underbrace{\frac{\sqrt{E_{s r} E_{r d}}}{\eta} \sum_{n=1}^{N}\left(h_{s r, 2 n}^{k} h_{r d, n}^{k}\right)}_{\zeta_{2}^{k}} \underbrace{\sqrt{E_{s d}} h_{s d, 1}^{k}}_{\zeta_{3}^{k}} \underbrace{\sqrt{E_{s d}} h_{s d, 2}^{k}}_{\zeta_{4}^{k}}]
$$

For the destination signal modeling, let us assume that the perfect D-QOSTBC is transmitted referring to Fig. 1b. In the followings, we can easily obtain the received signals at $D$ during the $k$ th block time as

$$
\begin{aligned}
& y_{1}^{k}=\zeta_{1}^{k} s_{1}^{k}+\zeta_{2}^{k} s_{2}^{k}+\zeta_{3}^{k} s_{3}^{k}+\zeta_{4}^{k} s_{4}^{k}+n_{1}^{k} \\
& y_{2}^{k}=-\zeta_{1}^{k} s_{2}^{k *}+\zeta_{2}^{k} s_{1}^{k *}-\zeta_{3}^{k} s_{4}^{k *}+\zeta_{4}^{k} s_{3}^{k *}+n_{2}^{k} \\
& y_{3}^{k}=\zeta_{1}^{k} s_{3}^{k}+\zeta_{2}^{k} s_{4}^{k}+\zeta_{3}^{k} s_{1}^{k}+\zeta_{4}^{k} s_{2}^{k}+n_{3}^{k} \\
& y_{4}^{k}=-\zeta_{1}^{k} s_{4}^{k *}+\zeta_{2}^{k} s_{3}^{k *}-\zeta_{3}^{k} s_{2}^{k *}+\zeta_{4}^{k} s_{1}^{k *}+n_{4}^{k} .
\end{aligned}
$$

Furthermore, (6) can be rewritten in matrix form as

$$
\mathbf{y}^{k}=\mathbf{h}^{k} \mathbf{S}^{k}+\mathbf{n}^{k}
$$

where $\mathbf{y}^{k}=\left[y_{1}^{k}, y_{2}^{k}, y_{3}^{k}, y_{4}^{k}\right]$ and $\mathbf{h}^{k}$ is the effective channel vector defined in (5). The effective noise is $\mathbf{n}^{k}=\left[n_{1}^{k}, n_{2}^{k}, n_{3}^{k}, n_{4}^{k}\right]$, and the symbol matrix $\mathbf{S}^{k}$ is

$$
\mathbf{S}^{k}=\left[\begin{array}{cccc}
s_{1}^{k} & -s_{2}^{k *} & s_{3}^{k} & -s_{4}^{k *} \\
s_{2}^{k} & s_{1}^{k *} & s_{4}^{k} & s_{3}^{k *} \\
s_{3}^{k} & -s_{4}^{k *} & s_{1}^{k} & -s_{2}^{k *} \\
s_{4}^{k} & s_{3}^{k *} & s_{2}^{k} & s_{1}^{k *}
\end{array}\right]
$$

where $(\cdot)^{*}$ denotes conjugate operation.

From (6), it is noted that the traditional D-QOSTBC transmission can be readily extended to a distributed fashion similar to $4 \times 1$ multi-input single-output point to point communication in single frequency network.

\subsection{Transmission Procedures with Protocol I}

The received signals during Time-slot 2 of Protocol I are same as (6). On the other hands, Protocol I provides additional information in every Time-slot 1. We will investigate those additional signal by exploiting the proposed robust STBC-like subsystems in the next section. Let us consider the additional received signal $y_{d}^{k}$ which is transmitted from $S$ to $D$ during Time-slot 1:

$$
y_{d, 1}^{k}=\sqrt{E_{s d}} h_{s d, 1}^{k} s_{1}^{k}+\sqrt{E_{s d}} h_{s d, 2}^{k} s_{2}^{k}+n_{D 1,1}^{k}
$$

where $E_{s d}$ denotes the average power available at $D$. Similarly to (1), $h_{s d, i}^{k}$ is the channel gain between the $i$ th transmit antenna of $S$ and $D$, and $n_{D 1,1}^{k}$ is an AWGN with 
$\mathcal{C N} \sim\left(0, \sigma_{n}^{2}\right)$. Assuming that the complete D-QOSTBC codes are transmitted, the additional signals of (9) can be expressed as

$$
\begin{aligned}
& y_{d, 1}^{k}=\sqrt{E_{s d}} h_{s d, 1}^{k} s_{1}^{k}+\sqrt{E_{s d}} h_{s d, 2}^{k} s_{2}^{k}+n_{D 1,1}^{k} \\
& y_{d, 2}^{k}=-\sqrt{E_{s d}} h_{s d, 1}^{k} s_{2}^{k *}+\sqrt{E_{s d}} h_{s d, 2}^{k} s_{1}^{k *}+n_{D 1,2}^{k} \\
& y_{d, 3}^{k}=\sqrt{E_{s d}} h_{s d, 1}^{k} s_{3}^{k}+\sqrt{E_{s d}} h_{s d, 2}^{k} s_{4}^{k}+n_{D 1,3}^{k} \\
& y_{d, 4}^{k}=-\sqrt{E_{s d}} h_{s d, 1}^{k} s_{4}^{k *}+\sqrt{E_{s d}} h_{s d, 2}^{k} s_{3}^{k *}+n_{D 1,4}^{k}
\end{aligned}
$$

We can see that (10) does not have all elements of QOSTBC block, and it merely have path coefficients $h_{s d, 1}^{k}$ and $h_{s d, 2}^{k}$.

\section{Destination Structure for Efficient DD-QOSTBC System}

In the following, we discuss differential decoding based on the conventional subsystem based on [10]. With simple manipulations of $y_{1}^{k}, y_{2}^{k}, y_{3}^{k}$ and $y_{4}^{k}$, we obtain

$$
\begin{aligned}
y_{1}^{k}+y_{3}^{k}= & \left(\zeta_{1}^{k}+\zeta_{3}^{k}\right)\left(s_{1}^{k}+s_{3}^{k}\right) \\
& +\left(\zeta_{2}^{k}+\zeta_{4}^{k}\right)\left(s_{2}^{k}+s_{4}^{k}\right)+n_{1}^{k}+n_{3}^{k} \\
y_{2}^{k}+y_{4}^{k}= & -\left(\zeta_{1}^{k}+\zeta_{3}^{k}\right)\left(s_{2}^{k *}+s_{4}^{k *}\right) \\
& +\left(\zeta_{2}^{k}+\zeta_{4}^{k}\right)\left(s_{1}^{k *}+s_{3}^{k *}\right)+n_{2}^{k}+n_{4}^{k} \\
y_{1}^{k}-y_{3}^{k}= & \left(\zeta_{1}^{k}-\zeta_{3}^{k}\right)\left(s_{1}^{k}-s_{3}^{k}\right) \\
& +\left(\zeta_{2}^{k}-\zeta_{4}^{k}\right)\left(s_{2}^{k}-s_{4}^{k}\right)+n_{1}^{k}-n_{3}^{k} \\
y_{2}^{k}-y_{4}^{k}= & -\left(\zeta_{1}^{k}-\zeta_{3}^{k}\right)\left(s_{2}^{k *}-s_{4}^{k *}\right) \\
& +\left(\zeta_{2}^{k}-\zeta_{4}^{k}\right)\left(s_{1}^{k *}-s_{3}^{k *}\right)+n_{2}^{k}-n_{4}^{k} .
\end{aligned}
$$

From (11), we can make two equivalent subsystems as follows

$$
\begin{aligned}
\mathbf{y}_{C 1}^{k} & =\mathbf{h}_{1}^{k} \mathbf{S}_{1}^{k}+\mathbf{n}_{1}^{k} \\
\mathbf{y}_{C 2}^{k} & =\mathbf{h}_{2}^{k} \mathbf{S}_{2}^{k}+\mathbf{n}_{2}^{k},
\end{aligned}
$$

where we define subscripts $C 1$ and $C 2$ as the first and second conventional subsystems, respectively. Effective vectors are represented as $\mathbf{y}_{C 1}^{k}=\left[y_{1}^{k}+y_{3}^{k}, y_{2}^{k}+y_{4}^{k}\right], \mathbf{y}_{C 2}^{k}=\left[y_{1}^{k}-y_{3}^{k}\right.$, $\left.y_{2}^{k}-y_{4}^{k}\right], \quad \mathbf{h}_{1}^{k}=\left[\zeta_{1}^{k}+\zeta_{3}^{k}, \zeta_{2}^{k}+\zeta_{4}^{k}\right], \quad \mathbf{h}_{2}^{k}=\left[\zeta_{1}^{k}-\zeta_{3}^{k}, \zeta_{2}^{k}-\zeta_{4}^{k}\right], \quad \mathbf{n}_{1}^{k}=\left[n_{1}^{k}+n_{3}^{k}, n_{2}^{k}+n_{4}^{k}\right], \quad$ and $\mathbf{n}_{2}^{k}=\left[n_{1}^{k}-n_{3}^{k}, n_{2}^{k}-n_{4}^{k}\right]$. $\mathbf{S}_{1}^{k}$ and $\mathbf{S}_{2}^{k}$ are defined as

$$
\begin{aligned}
& \mathbf{S}_{1}^{k}=\left[\begin{array}{cc}
s_{1}^{k}+s_{3}^{k} & -\left(s_{2}^{k *}+s_{4}^{k *}\right) \\
s_{2}^{k}+s_{4}^{k} & s_{1}^{k *}+s_{3}^{k *}
\end{array}\right] \\
& \mathbf{S}_{2}^{k}=\left[\begin{array}{cc}
s_{1}^{k}-s_{3}^{k} & -\left(s_{2}^{k *}-s_{4}^{k *}\right) \\
s_{2}^{k}-s_{4}^{k} & s_{1}^{k *}-s_{3}^{k *}
\end{array}\right] .
\end{aligned}
$$




\subsection{Proposed Robust STBC-Like Subsystems}

\subsubsection{Robust STBC-Like Subsystems for Protocol III}

Let us define the proposed robust STBC-like subsystems by mapping $\mathbf{y}_{C 1}^{k}$ to STBC-like format [9]:

$$
\mathbf{Y}_{1}^{k}=\mathbf{H}_{1}^{k} \mathbf{S}_{1}^{k}+\mathbf{N}_{1}^{k}
$$

where $\mathbf{S}_{1}^{k}$ is defined in (13). The matrices of $\mathbf{Y}_{1}^{k}, \mathbf{H}_{1}^{k}$, and $\mathbf{N}_{1}^{k}$ are

$$
\begin{aligned}
\mathbf{y}_{C 1}^{k} \mapsto \mathbf{Y}_{1}^{k} & =\left[\begin{array}{cc}
y_{1}^{k}+y_{3}^{k} & y_{2}^{k}+y_{4}^{k} \\
-\left(y_{2}^{k *}+y_{4}^{k *}\right) & y_{1}^{k *}+y_{3}^{k *}
\end{array}\right] \\
\mathbf{h}_{1}^{k} \mapsto \mathbf{H}_{1}^{k} & =\left[\begin{array}{cc}
\zeta_{1}^{k}+\zeta_{3}^{k} & \zeta_{2}^{k}+\zeta_{4}^{k} \\
-\left(\zeta_{2}^{k *}+\zeta_{4}^{k *}\right) & \zeta_{1}^{k *}+\zeta_{3}^{k *}
\end{array}\right] \\
\mathbf{n}_{1}^{k} \mapsto \mathbf{N}_{1}^{k} & =\left[\begin{array}{cc}
n_{1}^{k}+n_{3}^{k} & n_{2}^{k}+n_{4}^{k} \\
-\left(n_{2}^{k *}+n_{4}^{k *}\right) & n_{1}^{k *}+n_{3}^{k *}
\end{array}\right],
\end{aligned}
$$

where $\mapsto$ stands for mapping function. Similarly, the second robust STBC-like subsystem can be formulated by reformulating (11) with $\left(y_{1}^{k}-y_{3}^{k}\right)$ and $\left(y_{2}^{k}-y_{4}^{k}\right)$ of $\mathbf{y}_{C 2}^{k}$ as follows

$$
\mathbf{Y}_{2}^{k}=\mathbf{H}_{2}^{k} \mathbf{S}_{2}^{k}+\mathbf{N}_{2}^{k}
$$

where $\mathbf{S}_{2}^{k}$ is defined in (13), the matrices of $\mathbf{Y}_{2}^{k}, \mathbf{H}_{2}^{k}$, and $\mathbf{N}_{2}^{k}$ are

$$
\begin{aligned}
& \mathbf{y}_{C 2}^{k} \mapsto \mathbf{Y}_{2}^{k}=\left[\begin{array}{cc}
y_{1}^{k}-y_{3}^{k} & y_{2}^{k}-y_{4}^{k} \\
-\left(y_{2}^{k *}-y_{4}^{k *}\right) & y_{1}^{k *}-y_{3}^{k *}
\end{array}\right] \\
& \mathbf{h}_{2}^{k} \mapsto \mathbf{H}_{2}^{k}= {\left[\begin{array}{cc}
\zeta_{1}^{k}-\zeta_{3}^{k} & \zeta_{2}^{k}-\zeta_{4}^{k} \\
-\left(\zeta_{2}^{k *}-\zeta_{4}^{k *}\right) & \zeta_{1}^{k *}-\zeta_{3}^{k *}
\end{array}\right] } \\
& \mathbf{n}_{2}^{k} \mapsto \mathbf{N}_{2}^{k}=\left[\begin{array}{cc}
n_{1}^{k}-n_{3}^{k} & n_{2}^{k}-n_{4}^{k} \\
-\left(n_{2}^{k *}-n_{4}^{k *}\right) & n_{1}^{k *}-n_{3}^{k *}
\end{array}\right] .
\end{aligned}
$$

From (14) and (16), it is noticed that both $\left(\mathbf{H}_{1}^{k}, \mathbf{H}_{2}^{k}\right)$ and $\left(\mathbf{S}_{1}^{k}, \mathbf{S}_{2}^{k}\right)$ matrices preserve the orthogonal property, which is important for the efficient differential decoding of (29) and (30). The equivalent property between the DD-QOSTBC system and robust STBC-like subsystems are readily proven, by extracting sequentially $(1,1)$ and $(1,2)$ elements of $\mathbf{B}_{1}^{k}$ and $\mathbf{B}_{2}^{k}$ which are defined as $\mathbf{B}_{1}^{k}=\left(\mathbf{Y}_{1}^{k}+\mathbf{Y}_{2}^{k}\right) / 2$ and $\mathbf{B}_{2}^{k}=\left(\mathbf{Y}_{1}^{k}-\mathbf{Y}_{2}^{k}\right) / 2$.

\subsubsection{Robust STBC-Like Subsystems for Protocol I}

Here, it is assumed that manipulation for robust STBC-like subsystem is also performed with the additional information of (10). We can also observe the equivalent property from the signal (10) in every Time-slot 1 . With manipulations of $y_{d, 1}^{k}, y_{d, 2}^{k}, y_{d, 3}^{k}$ and $y_{d, 4}^{k}$, the first additional robust STBC-like subsystem can be expressed as

$$
\mathbf{Y}_{a, 1}^{k}=\mathbf{H}_{a}^{k} \mathbf{S}_{1}^{k}+\mathbf{N}_{a, 1}^{k},
$$


where $\mathbf{S}_{1}^{k}$ is expressed in (13). The matrices of $\mathbf{Y}_{a, 1}^{k}, \mathbf{H}_{a}^{k}$, and $\mathbf{N}_{a, 1}^{k}$ are defined, respectively, as

$$
\begin{aligned}
\mathbf{Y}_{a, 1}^{k}= & {\left[\begin{array}{cc}
y_{d, 1}^{k}+y_{d, 3}^{k} & y_{d, 2}^{k}+y_{d, 4}^{k} \\
-\left(y_{d, 2}^{k *}+y_{d, 4}^{k *}\right) & y_{d, 1}^{k *}+y_{d, 3}^{k *}
\end{array}\right] } \\
\mathbf{H}_{a}^{k}= & {\left[\begin{array}{cc}
\sqrt{E_{s d}} h_{s d, 1}^{k} & \sqrt{E_{s d}} h_{s d, 2}^{k} \\
-\sqrt{E_{s d}} h_{s d, 2}^{k *} & \sqrt{E_{s d}} h_{s d, 1}^{k *}
\end{array}\right] } \\
\mathbf{N}_{a, 1}^{k}= & {\left[\begin{array}{cc}
n_{D 1,1}^{k}+n_{D 1,3}^{k} & n_{D 1,2}^{k}+n_{D 1,4}^{k} \\
-\left(n_{D 1,2}^{k *}+n_{D 1,4}^{k *}\right) & n_{D 1,1}^{k *}+n_{D 1,3}^{k *}
\end{array}\right] . }
\end{aligned}
$$

The second robust STBC-like subsystem is similarly given by

$$
\mathbf{Y}_{a, 2}^{k}=\mathbf{H}_{a}^{k} \mathbf{S}_{2}^{k}+\mathbf{N}_{a, 2}^{k}
$$

where $\mathbf{S}_{2}^{k}$ is expressed in (13). It is noticed that $\mathbf{H}_{a}^{k}$ is identically defined as in (19) while $\mathbf{Y}_{a, 2}^{k}$ and $\mathbf{N}_{a, 2}^{k}$ are

$$
\begin{aligned}
\mathbf{Y}_{a, 2}^{k}= & {\left[\begin{array}{cc}
y_{d, 1}^{k}-y_{d, 3}^{k} & y_{d, 2}^{k}-y_{d, 4}^{k} \\
-\left(y_{d, 2}^{k *}-y_{d, 4}^{k *}\right) & y_{d, 1}^{k *}-y_{d, 3}^{k *}
\end{array}\right] } \\
\mathbf{N}_{a, 2}^{k}= & {\left[\begin{array}{cc}
n_{D 1,1}^{k}-n_{D 1,3}^{k} & n_{D 1,2}^{k}-n_{D 1,4}^{k} \\
-\left(n_{D 1,2}^{k *}-n_{D 1,4}^{k *}\right) & n_{D 1,1}^{k *}-n_{D 1,3}^{k *}
\end{array}\right] . }
\end{aligned}
$$

From (18) to (21), the effective channel matrix $\mathbf{H}_{a}^{k}$ only contains the channels of $S \rightarrow D$ link, while effective channel matrices $\left(\mathbf{H}_{1}^{k}, \mathbf{H}_{2}^{k}\right)$ contain the channels of both $S \rightarrow D$ and $S \rightarrow R \rightarrow D$ links. Therefore, $\mathbf{H}_{a}^{k}$ can not provide cooperative diversity. However, the advantage of our subsystem is that the surplus signals $\mathbf{Y}_{a, 1}^{k}$ and $\mathbf{Y}_{a, 2}^{k}$ can be combined with $\mathbf{Y}_{1}^{k}$ and $\mathbf{Y}_{2}^{k}$ by multiplying weighting factors, since $\left(\mathbf{Y}_{1}^{k}, \mathbf{Y}_{2}^{k}\right)$ and $\left(\mathbf{Y}_{a, 1}^{k}, \mathbf{Y}_{a, 2}^{k}\right)$ have the same shapes. We define those shapes as matrix-wise sets.

\section{Differential Encoding and Decoding for DD-QOSTBC System}

In this section, we present the efficient DD-QOSTBC encoding and decoding procedures for the aforementioned robust STBC-like subsystems.

\subsection{DD-QOSTBC Encoding}

In Fig. 1a, differential encoding for each symbol is equivalently treated in the first robust STBC-like subsystems of (14) and (16). Therefore, we can apply the general D-STBC encoding rule to these two subsystems as

$$
\mathbf{S}_{1}^{k}=\frac{1}{u_{1}^{k-1}} \mathbf{S}_{1}^{k-1} \mathbf{X}_{1}^{k}
$$

where $u_{1}^{k-1}=\sqrt{\left|x_{1}^{k-1}+x_{3}^{k-1}\right|^{2}+\left|x_{2}^{k-1}+x_{4}^{k-1}\right|^{2}}$ is a normalization factor. The transmit symbol matrix of $\mathbf{X}_{1}^{k}$ is defined as 


$$
\mathbf{X}_{1}^{k}=\left[\begin{array}{cc}
x_{1}^{k}+x_{3}^{k} & -\left(x_{2}^{k *}+x_{4}^{k *}\right) \\
x_{2}^{k}+x_{4}^{k} & x_{1}^{k *}+x_{3}^{k *}
\end{array}\right],
$$

where the linear combination rule of data symbols $x_{k}^{k}$ (for $k=1,2,3,4$ ) follows the formulation rule of (11). $\left(x_{1}^{k}, x_{2}^{k}\right)$ are the cardinality of phase-shift-keying (PSK) constellation set $\mathcal{V}_{1}$, i.e., $x_{1}, x_{2} \in \mathcal{V}_{1}$. In contrast, $\left(x_{3}^{k}, x_{4}^{k}\right)$ are the rotated (offset) cardinality of the PSK constellation set $\mathcal{V}_{2}$, i.e., $x_{3}, x_{4} \in \mathcal{V}_{2}$, and these two constellation sets are defined to prevent the sum of elements of $\mathbf{X}_{1}^{k}$ becoming a zero matrix [10]. Similarly with (22), the differential encoding for the second robust STBC-like subsystem for (18) and (20) can be expressed as

$$
\mathbf{S}_{2}^{k}=\frac{1}{u_{2}^{k-1}} \mathbf{S}_{2}^{k-1} \mathbf{X}_{2}^{k}
$$

where $u_{2}^{k-1}=\sqrt{\left|x_{1}^{k-1}-x_{3}^{k-1}\right|^{2}+\left|x_{2}^{k-1}-x_{4}^{k-1}\right|^{2}}$ and the transmitted symbol matrix $\mathbf{X}_{2}^{k}$ is given by

$$
\mathbf{X}_{2}^{k}=\left[\begin{array}{cc}
x_{1}^{k}-x_{3}^{k} & -\left(x_{2}^{k *}-x_{4}^{k *}\right) \\
x_{2}^{k}-x_{4}^{k} & x_{1}^{k *}-x_{3}^{k *}
\end{array}\right]
$$

\subsection{DD-QOSTBC Decoding for Protocol III}

We present the proposed ML-based differential decoding scheme with no CSI. It is assumed that the channels are constant over the transmission of the two consecutive block time, i.e., $\mathbf{H}_{1}^{k-1}=\mathbf{H}_{1}^{k}, \mathbf{H}_{2}^{k-1}=\mathbf{H}_{2}^{k}$ and $\mathbf{H}_{a}^{k-1}=\mathbf{H}_{a}^{k}$. For the comprehensive understanding, we first consider the differential decoding for $\mathbf{Y}_{1}^{k}$ and $\mathbf{Y}_{2}^{k}$ (see (14) and (16)), where $\mathbf{S}_{1}^{k}$ and $\mathbf{S}_{2}^{k}$ are encoded according to the rules of (22) and (24). With the equivalent property between the DD-QOSTBC system and two robust STBC-like subsystems, the proposed ML-based differential decoding which eliminates the necessity of CSI is defined as

$$
\begin{aligned}
& \hat{\mathbf{X}}_{P 3,12}^{k}=\underset{x_{1}, x_{2} \in \mathcal{V}_{1}}{\operatorname{argmin}}\left\|\mathbf{V}_{1}^{k}-\frac{1}{2}\left(\mathbf{Y}_{1}^{(k-1) \dagger} \mathbf{Y}_{1}^{k}+\mathbf{Y}_{2}^{(k-1) \dagger} \mathbf{Y}_{2}^{k}\right)\right\|^{2} \\
& \hat{\mathbf{X}}_{P 3,34}^{k}=\underset{x_{3}, x_{4} \in \mathcal{V}_{2}}{\operatorname{argmin}}\left\|\mathbf{V}_{2}^{k}-\frac{1}{2}\left(\mathbf{Y}_{1}^{(k-1) \dagger} \mathbf{Y}_{1}^{k}-\mathbf{Y}_{2}^{(k-1) \dagger} \mathbf{Y}_{2}^{k}\right)\right\|^{2},
\end{aligned}
$$

where the reference symbol matrices $\mathbf{V}_{1}^{k}$ and $\mathbf{V}_{2}^{k}$ are

$$
\mathbf{V}_{1}^{k}=\left[\begin{array}{cc}
c_{1}^{k} & -c_{2}^{k *} \\
c_{2}^{k} & c_{1}^{k *}
\end{array}\right], \quad \mathbf{V}_{2}^{k}=\left[\begin{array}{cc}
c_{3}^{k} & -c_{4}^{k *} \\
c_{4}^{k} & c_{3}^{k *}
\end{array}\right]
$$

Here, it is noticed that the correlation between $\mathbf{H}_{i}^{(k-1)}$ and $\mathbf{H}_{i}^{k}$ can be perfectly decoupled from the orthogonal properties as 


$$
\begin{aligned}
\left\|\mathbf{H}_{1}^{k}\right\|^{2} & =\mathbf{H}_{1}^{(k-1) \dagger} \mathbf{H}_{1}^{k} \\
& =\left(\left(\zeta_{1}^{k}+\zeta_{3}^{k}\right)^{2}+\left(\zeta_{2}^{k}+\zeta_{4}^{k}\right)^{2}\right) \mathbf{I}_{2}=\left(\beta_{1}^{k}\right)^{2} \mathbf{I}_{2} \\
\left\|\mathbf{H}_{2}^{k}\right\|^{2} & =\mathbf{H}_{2}^{(k-1) \dagger} \mathbf{H}_{2}^{k} \\
& =\left(\left(\zeta_{1}^{k}-\zeta_{3}^{k}\right)^{2}+\left(\zeta_{2}^{k}-\zeta_{4}^{k}\right)^{2}\right) \mathbf{I}_{2}=\left(\beta_{2}^{k}\right)^{2} \mathbf{I}_{2} .
\end{aligned}
$$

The channel gains in (28) become real value, thus (26) can be rewritten as

$$
\begin{gathered}
\hat{\mathbf{X}}_{P 3,12}^{k}=\underset{x_{1}, x_{2} \in \mathcal{V}_{1}}{\operatorname{argmin}}\left[\begin{array}{ll}
\left|c_{1}^{k}-\left(\lambda^{k} x_{1}^{k}+\dot{n}_{1}^{k}\right)\right|^{2} & \star \\
\left|c_{2}^{k}-\left(\lambda^{k} x_{2}^{k}+\dot{n}_{2}^{k}\right)\right|^{2} & \star
\end{array}\right] \\
\hat{\mathbf{X}}_{P 3,34}^{k}=\underset{x_{3}, x_{4} \in \mathcal{V}_{2}}{\operatorname{argmin}}\left[\begin{array}{ll}
\left|c_{3}^{k}-\left(\lambda^{k} x_{3}^{k}+\dot{n}_{3}^{k}\right)\right|^{2} & \star \\
\left|c_{4}^{k}-\left(\lambda^{k} x_{4}^{k}+\dot{n}_{4}^{k}\right)\right|^{2} & \star
\end{array}\right],
\end{gathered}
$$

where $\lambda^{k}=\frac{\left(\beta_{1}^{k}\right)^{2} u_{2}^{k-1}+\left(\beta_{2}^{k}\right)^{2} u_{1}^{k-1}}{2 u_{1}^{k-1} u_{2}^{k-1}}$. ' $\star$ ' means 'this element does not need to be involved in decoding', that this notation is caused by the manipulation of left column ${ }^{1}$. The noise terms $\dot{n}_{1}^{k}, \dot{n}_{2}^{k}, \dot{n}_{3}^{k}, \quad$ and $\quad \dot{n}_{4}^{k} \quad$ are $\quad \dot{n}_{1}^{k}=\frac{1}{2}\left(s_{1}^{k-1}\left(\beta_{1}^{k-1}\right)^{2} n_{1}^{k}+n_{1}^{(k-1) *}\left(\beta_{1}^{k}\right)^{2} s_{1}^{k}+\sigma_{n}^{2}\right), \quad \dot{n}_{2}^{k}=\frac{1}{2}\left(s_{2}^{k-1}\right.$ $\left.\left(\beta_{1}^{k-1}\right)^{2} n_{2}^{k}+n_{2}^{(k-1) *}\left(\beta_{1}^{k}\right)^{2} s_{2}^{k}+\sigma_{n}^{2}\right), \quad \dot{n}_{3}^{k}=\frac{1}{2}\left(s_{3}^{k-1}\left(\beta_{2}^{k-1}\right)^{2} n_{3}^{k}+n_{3}^{(k-1) *}\left(\beta_{2}^{k}\right)^{2} s_{3}^{k}+\sigma_{n}^{2}\right), \quad$ and $\dot{n}_{4}^{k}=\frac{1}{2}\left(s_{4}^{k-1}\left(\beta_{2}^{k-1}\right)^{2} n_{4}^{k}+n_{4}^{(k-1) *}\left(\beta_{2}^{k}\right)^{2} s_{4}^{k}+\sigma_{n}^{2}\right)$, respectively.

$$
\left.\begin{array}{rl}
\hat{\mathbf{X}}_{P 1,12}^{k}= & \underset{x_{1}, x_{2} \in \mathcal{V}_{1}}{\operatorname{argmin}} \| \mathbf{V}_{1}^{k}-\frac{1}{2}\left(\left(1-\omega^{k}\right)\left(\mathbf{Y}_{1}^{(k-1) \dagger} \mathbf{Y}_{1}^{k}+\mathbf{Y}_{2}^{(k-1) \dagger} \mathbf{Y}_{2}^{k}\right)\right. \\
& \left.+\omega^{k}\left(\mathbf{Y}_{a, 1}^{(k-1) \dagger} \mathbf{Y}_{a, 1}^{k}+\mathbf{Y}_{a, 2}^{(k-1) \dagger} \mathbf{Y}_{a, 2}^{k}\right)\right) \|^{2} \\
= & \underset{x_{1}, x_{2} \in \mathcal{V}_{1}}{\operatorname{argmin}}\left[\left|c_{1}^{k}-\left(\frac{\left(1-w^{k}\right)\left(\left(\beta_{1}^{k}\right)^{2}+\left(\beta_{3}^{k}\right)^{2}\right) u_{2}^{k-1}+w^{k}\left(\left(\beta_{2}^{k}\right)^{2}+\left(\beta_{3}^{k}\right)^{2}\right) u_{1}^{k-1}}{2 u_{1}^{k-1} u_{2}^{k-1}+\ddot{n}_{1}^{k}}\right)\right|^{2} \star\right. \\
c_{2}^{k}-\left.\left(\frac{\left(1-w^{k}\right)\left(\left(\beta_{1}^{k}\right)^{2}+\left(\beta_{3}^{k}\right)^{2}\right) u_{2}^{k-1}+w^{k}\left(\left(\beta_{2}^{k}\right)^{2}+\left(\beta_{3}^{k}\right)^{2}\right) u_{1}^{k-1}}{2 u_{1}^{k-1} u_{2}^{k-1}} x_{2}^{k}+\ddot{n}_{2}^{k}\right)\right|^{2} \star
\end{array}\right]
$$

1 For example, the STBC matrix $\left[\begin{array}{l}a \star \\ b \star\end{array}\right] \triangleq\left[\begin{array}{l}a-b^{*} \\ b a^{*}\end{array}\right]$, since second column can be constructed from first column $a$ and $b$. We do not need to consider second column when detection is performed. 


$$
\begin{aligned}
& \hat{\mathbf{X}}_{P 1,34}^{k}= \underset{x_{3}, x_{4} \in \mathcal{V}_{2}}{\operatorname{argmin}} \| \mathbf{V}_{2}^{k}-\frac{1}{2}\left(\left(1-\omega^{k}\right)\left(\mathbf{Y}_{1}^{(k-1) \dagger} \mathbf{Y}_{1}^{k}-\mathbf{Y}_{2}^{(k-1) \dagger} \mathbf{Y}_{2}^{k}\right)\right. \\
&\left.+\omega^{k}\left(\mathbf{Y}_{a, 1}^{(k-1) \dagger} \mathbf{Y}_{a, 1}^{k}-\mathbf{Y}_{a, 2}^{(k-1) \dagger} \mathbf{Y}_{a, 2}^{k}\right)\right) \|^{2} \\
&= \underset{x_{3}, x_{4} \in \mathcal{V}_{2}}{\operatorname{argmin}}\left[\left|c_{3}^{k}-\left(\frac{\left(1-w^{k}\right)\left(\left(\beta_{1}^{k}\right)^{2}+\left(\beta_{3}^{k}\right)^{2}\right) u_{2}^{k-1}+w^{k}\left(\left(\beta_{2}^{k}\right)^{2}+\left(\beta_{3}^{k}\right)^{2}\right) u_{1}^{k-1}}{2 u_{1}^{k-1} u_{2}^{k-1}+\ddot{n}_{3}^{k}}\right)\right|^{2} \star\right. \\
& c_{4}^{k}-\left.\left(\frac{\left(1-w^{k}\right)\left(\left(\beta_{1}^{k}\right)^{2}+\left(\beta_{3}^{k}\right)^{2}\right) u_{2}^{k-1}+w^{k}\left(\left(\beta_{2}^{k}\right)^{2}+\left(\beta_{3}^{k}\right)^{2}\right) u_{1}^{k-1}}{2 u_{1}^{k-1} u_{2}^{k-1}} x_{4}^{k}+\ddot{n}_{4}^{k}\right)\right|^{2} \star \star
\end{aligned}
$$

\subsection{DD-QOSTBC Decoding for Protocol I (Joint Suboptimal Differential Decoding)}

From (29) and (30), we present the proposed JSDD which combines the matrix-wise outputs $\left(\mathbf{Y}_{1}^{k}, \mathbf{Y}_{2}^{k}\right)$ and $\left(\mathbf{Y}_{a, 1}^{k}, \mathbf{Y}_{a, 2}^{k}\right)$ based on robust STBC-like subsystems. The overall JSDD procedures associated with the detection of $x_{1}^{k}, x_{2}^{k}, x_{3}^{k}$ and $x_{4}^{k}$ symbols, are represented as following steps.

1. The original symbols are detected from the outputs $\mathbf{Y}_{1}^{k}$ and $\mathbf{Y}_{2}^{k}$ by using (29) and (30).

2. Through a similar approach with step 1), the same symbols are also detected from the outputs $\mathbf{Y}_{a, 1}^{k}$ and $\mathbf{Y}_{a, 2}^{k}$ by using (29) and (30). Here, we obtain channel gains $\left\|\mathbf{H}_{a}^{k}\right\|^{2}=E_{s d}\left(\left(h_{s d, 1}^{k}\right)^{2}+\left(h_{s d, 2}^{k}\right)^{2}\right) \mathbf{I}_{2}=\left(\beta_{3}^{k}\right)^{2} \mathbf{I}_{2}$.

3. Calculate the sum of error distances in terms of $x_{1}^{k}, x_{2}^{k}, x_{3}^{k}$ and $x_{4}^{k}$ from $\left(\mathbf{Y}_{1}^{k}, \mathbf{Y}_{2}^{k}\right)$ and $\left(\mathbf{Y}_{a, 1}^{k}, \mathbf{Y}_{a, 2}^{k}\right)$. We can define relative weighting factor in (33).

4. As a result, the original symbols experiencing different links can be combined to improve the system performance. As a suboptimal approach, we consider the MLbased JSDD scheme as (31) and (32).

In (32), weighting factor is defined as

$$
w^{k}=\frac{\sum_{i=1}^{4}\left|c_{i}^{k}-2 y_{d, i}^{(k-1) \dagger} y_{d, i}^{k}\right|^{2}}{\sum_{i=1}^{4}\left(\left|c_{i}^{k}-2 y_{d, i}^{(k-1) \dagger} y_{d, i}^{k}\right|^{2}+\left|c_{i}^{k}-2 y_{i}^{(k-1) \dagger} y_{i}^{k}\right|^{2}\right)},
$$

and effective noise terms are $\ddot{n}_{1}^{k}=\left(1-w^{k}\right) \dot{n}_{1}^{k}+\frac{w^{k}}{2}\left(s_{1}^{k-1}\left(\beta_{3}^{k-1}\right)^{2} n_{D 1,1}^{k}+n_{D 1,1}^{(k-1) *}\left(\beta_{3}^{k}\right)^{2} s_{1}^{k}+\right.$ $\left.\sigma_{n}^{2}\right), \quad \ddot{n}_{2}^{k}=\left(1-w^{k}\right) \dot{n}_{2}^{k}+\frac{w^{k}}{2}\left(s_{2}^{k-1}\left(\beta_{3}^{k-1}\right)^{2} n_{D 1,2}^{k}+n_{D 1,2}^{(k-1) *}\left(\beta_{3}^{k}\right)^{2} s_{2}^{k}+\sigma_{n}^{2}\right), \quad \ddot{n}_{3}^{k}=\left(1-w^{k}\right) \dot{n}_{3}^{k}+$ $\frac{w^{k}}{2}\left(s_{3}^{k-1}\left(\beta_{3}^{k-1}\right)^{2} n_{D 1,3}^{k}+n_{D 1,3}^{(k-1) *}\left(\beta_{3}^{k}\right)^{2} s_{3}^{k}+\sigma_{n}^{2}\right)$, and $\ddot{n}_{4}^{k}=\left(1-w^{k}\right) \dot{n}_{4}^{k}+\frac{w^{k}}{2}\left(s_{4}^{k-1}\left(\beta_{3}^{k-1}\right)^{2} n_{D 1,4}^{k}\right.$ $\left.+n_{D 1,4}^{(k-1) *}\left(\beta_{3}^{k}\right)^{2} s_{4}^{k}+\sigma_{n}^{2}\right)$, respectively. 


\section{Performance Evaluations}

In this section, we accomplish the performance evaluations for the proposed DD-QOSTBC systems. For verifying the gain of the proposed system, we have considered various QOSTBC systems:

- DD-QOSTBC (P3): Protocol III equipped with the ML-based differential decoding of (26)

- DD-QOSTBC-JSDD (P1): Protocol I equipped with the proposed JSDD of (31) and (32)

- DD-QOSTBC-MRC (P1): Protocol I equipped with a (differential) maximum ratio combining (MRC) scheme

- DD-QOSTBC (Direct): Protocol I only considering the received differential signals during Time-slot 1

- DC-QOSTBC (P3)

- DC-QOSTBC-MRC (P1)

- DC-QOSTBC (Direct)

It is assumed that an identity matrix $\mathbf{I}_{2}$ is transmitted to initiate the DD-QOSTBC decoding in the beginning of transmission. It is also assumed that all of associated links are modeled as a Rayleigh channel. The frames consist of QPSK symbols, and a offset for $\mathcal{V}_{2}$ is $\pi / 4$. The number of $R, N$ is 2, and ML detection is performed. The symbol matrices $\mathbf{S}_{1}^{k}$ and $\mathbf{S}_{2}^{k}$ satisfy the power constraint $E\left[\left|s_{1}^{k}+s_{3}^{k}\right|^{2}+\left|s_{2}^{k}+s_{4}^{k}\right|^{2}\right]=E\left[\left|s_{1}^{k}-s_{3}^{k}\right|^{2}+\left|s_{2}^{k}-s_{4}^{k}\right|^{2}\right]=2$ and each symbol has unit variance.

Figure 2 depicts the bit-error-rate (BER) performances of various QOSTBC systems as a function of signal-to-noise (SNR), when $E_{s r} / \sigma_{n, R}=20 \mathrm{~dB}$. It is firstly observed that the DC-QOSTBC systems outperform the DD-QOSTBC systems, of which result can be expected from a traditional communication system [3]. It is found that the DD-QOSTBC (P1) systems outperform the DD-QOSTBC (P3) systems as SNR increases because Protocol I provides additional information during Time-slot 1. In addition, it is also found that the proposed DD-QOSTBC-JSDD (P1) outperforms than the DD-QOSTBC-MRC (P1),

Fig. 2 BER performances of various DD-QOSTBC systems as a function of SNR when $E_{s r} / \sigma_{n, R}=20 \mathrm{~dB}$

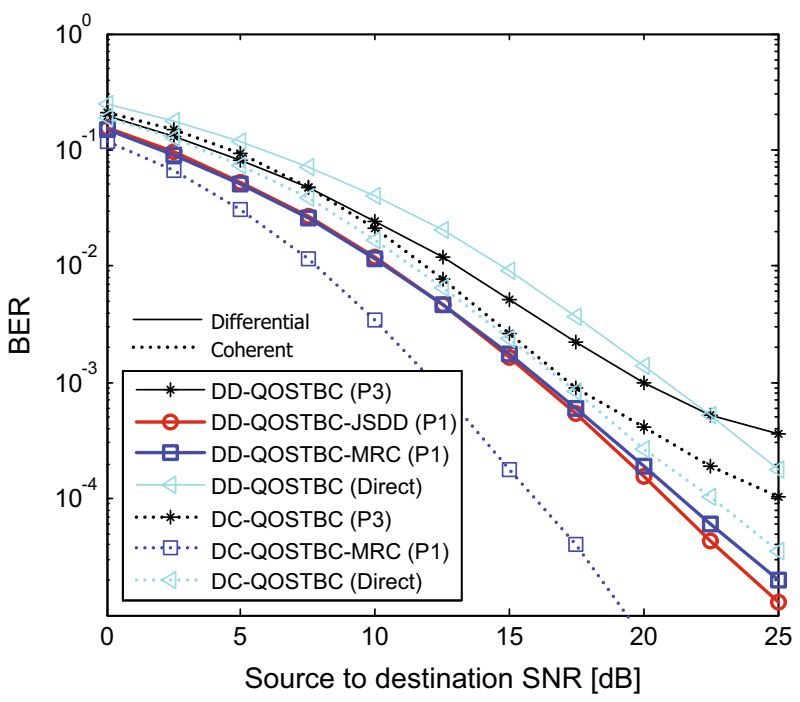


Fig. 3 BER performances of various DD-QOSTBC systems as a function of SNR when $E_{s r} / \sigma_{n, R}=5 \mathrm{~dB}$

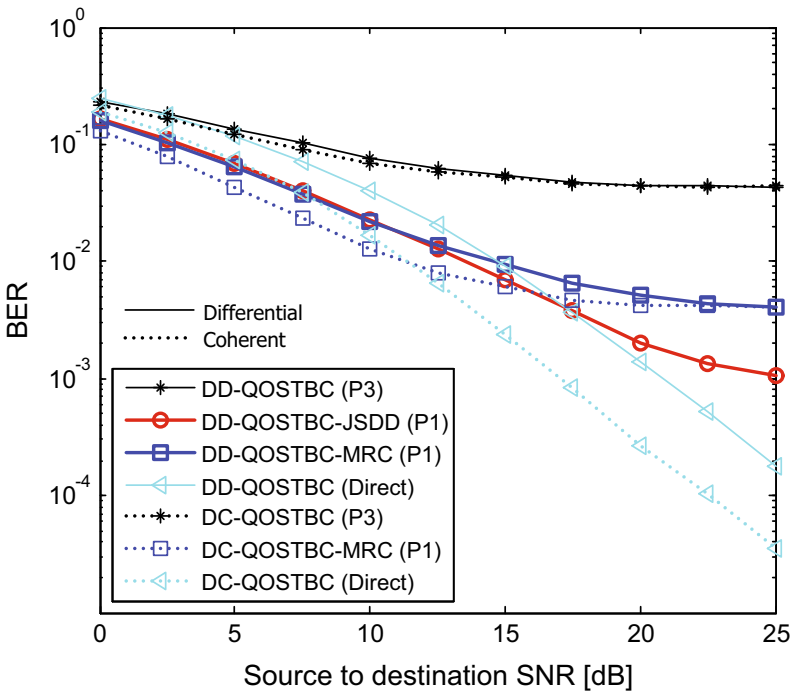

and this result reveals the robustness of the proposed JSDD scheme as well as its higher efficiency.

Figure 3 depicts the BER performances of QOSTBC systems as a function of signal-tonoise (SNR), when $E_{s r} / \sigma_{n, R}=5 \mathrm{~dB}$. The error floor effect is observed due to the limited SNR at $R$. As $S \rightarrow D$ does not affect from SNR of $R$, the performance of DC-QOSTBC (Direct) is same in both Figs. 2 and 3. This tendency is also observed in the DD-QOSTBC (Direct) case.

\section{Conclusions}

In this paper, we have presented the efficient robust DD-QOSTBC for multiple relay networks, especially the JSDD scheme for Protocol I. In addition, it is shown that the DDQOSTBC system can be readily applied to Protocol III. In the performance evaluations, we have verified the benefits of the proposed DD-QOSTBC systems by comparing with the DC-QOSTBC systems.

Acknowledgments This work was supported by the ICT R\&D program of MSIP/IITP (1391202006, A Study on Next Generation Interactive Terrestrial Broadcasting System). This research was also supported by the MSIP (Ministry of Science, ICT and Future Planning), Korea, under the ITRC (Information Technology Research Center) support program (IITP-2015-H8501-15-1001) supervised by the IITP (Institute for Information \& communications Technology Promotion).

Open Access This article is distributed under the terms of the Creative Commons Attribution 4.0 International License (http://creativecommons.org/licenses/by/4.0/), which permits unrestricted use, distribution, and reproduction in any medium, provided you give appropriate credit to the original author(s) and the source, provide a link to the Creative Commons license, and indicate if changes were made.

\section{References}

1. Kui, Z. F., Han, J. S., Choi, J. M., Jeon, S. H., Park, J. S., Oh, Y. H., et al. (2015). Advanced preamble transmit diversity of polarized DVB-T2 MISO system using hybrid differential modulation. IEEE Transactions on Broadcasting. doi:10.1109/TBC.2015.2465142. 
2. Han, J. S., Baek, J. S., \& Seo, J. S. (2013). MIMO-OFDM transceivers with dual-polarized division multiplexing and diversity for multimedia broadcasting services. IEEE Transactions on Broadcasting, 59(1), 174-182.

3. Wang, L., Li, L., Xu, C., Liang, D., Ng, S. X., \& Hanzo, L. (2014). Multiple-symbol joint signal processing for differentially encoded single-and multi-carrier communications: principles, designs and applications. IEEE Communications Surveys and Tutorials, 16(2), 689-712.

4. El Astal, M. T., Abu-Hudrouss, A. M., \& Olivier, J. C. (2014). Improved signal detection of wireless relaying networks employing space-time block codes under imperfect synchronization. Wireless Personal Communications, 82(1), 533-550.

5. Avendi, M. R., \& Jafarkhani, H. (2015). Differential distributed space-time coding with imperfect synchronization in frequency-selective channels. IEEE Transactions on Wireless Communications, 14(4), 1811-1822.

6. Han, J. S., Baek, J. S., Jeon, S. H., \& Seo, J. S. (2014). Cooperative networks with amplify-and-forward multiple-full-duplex relays. IEEE Transactions on Wireless Communications, 13(4), 2137-2147.

7. Lee, J. H. (2015). Cooperative relaying protocol for improving physical layer security in wireless decode-and-forward relaying networks. Wireless Personal Communications, 83(4), 3033-3044.

8. Zhao, Q., \& Li, H. (2007). Differential modulation for cooperative wireless systems. IEEE Transactions on Signal Processing, 55(5), 2273-2283.

9. Park, S. K., Han, J. S., Choi, J. M., \& Seo, J. S. (2014). Efficient differential quasi-orthogonal spacetime block coded system. IEICE Communications Express, 3(2), 347-351.

10. Zhu, Y., \& Jafarkhani, H. (2005). Differential modulation based on quasi-orthogonal codes. IEEE Transactions on Wireless Communications, 4(6), 3005-3017.

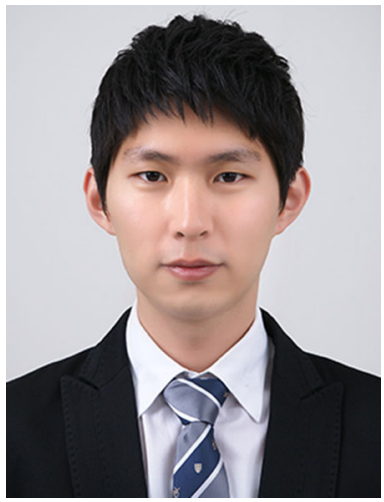

Soonki Jo received B.S. degree in electrical and electronic engineering from Yonsei University, Seoul, Korea, in 2009, and he is currently pursuing the Ph.D. degree in electrical and electronic engineering from Yonsei University, Seoul, Korea. His research interests include FBMC, MIMO systems, transmission strategies and wireless communication systems.

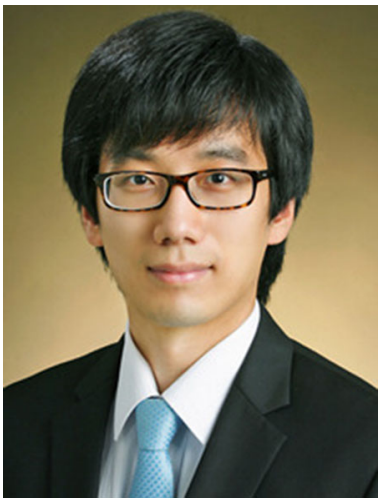

Jae-Shin Han received the B.S. degree in electronics engineering from Konkuk University, Seoul, Korea, in 2011. He is currently pursuing the combined Masters and Doctorate degree in electrical and electronic engineering with the Department of Electrical and Electronic Engineering, Yonsei University, Seoul. His current research interests include channel estimation, equalization, and full-duplex cooperative networks for broadband wireless communication systems. 

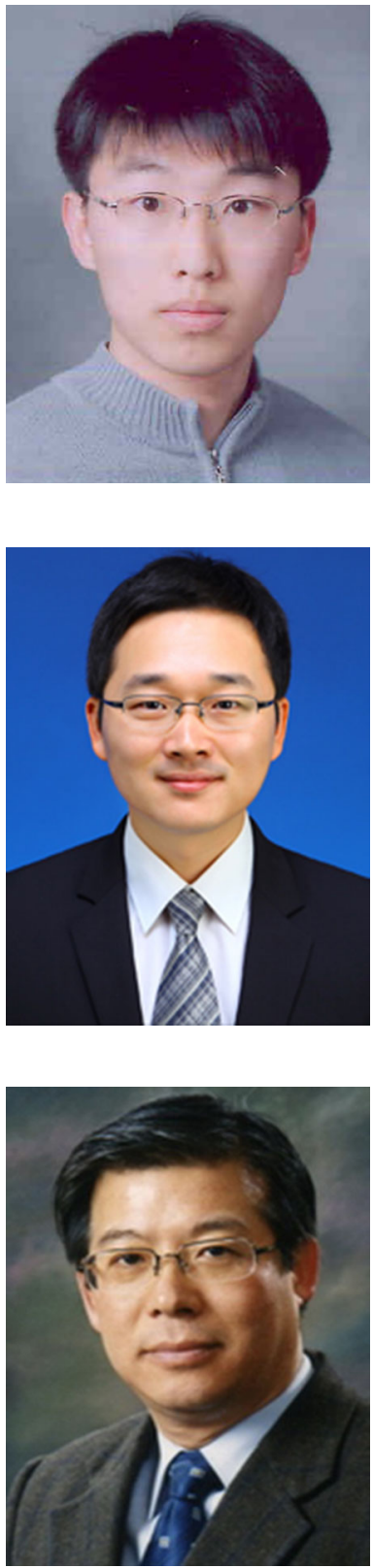

Sungho Jeon received the B.S. (with Honors) and M.S. degrees in electrical and electronics engineering from Yonsei University, Seoul, Korea, in August 2005 and February 2007, respectively. Since March 2007, he has been with the Technical Research Institute at Korean Broadcasting System (KBS), where he is currently a Research Engineer. From March 2011, he pursues a Ph.D. degree in electrical and electronics engineering with emphasis on 4K UHDTV terrestrial digital broadcasting systems at the Yonsei University.

Jeong-Min Choi received B.S. degree in electronic and information engineering from Seoul National University of Science and Technology, Seoul, Korea, in 2010, and the M.S. degree in electrical and electronic engineering from Yonsei University, Seoul, Korea, in 2012, respectively. Since 2012, he has been currently working toward the $\mathrm{Ph} . \mathrm{D}$. at the same university. His main research interests include signal processing for digital broadcasting and communication systems.

Jong-Soo Seo received the B.S. degree in electronics engineering from Yonsei University, Seoul, Korea, in 1975, and the M.S. and Ph.D. degrees from the University of Ottawa, Ottawa, ON, Canada, in 1983 and 1988, respectively. He was with IDC and CAL, Canada, engaged in research on digital satellite communications and data broadcasting systems for 6 years. Since 1995, he has been with the Department of Electrical and Electronic Engineering, Yonsei University, where he is currently a Professor. His current research interests include mobile multimedia broadcasting and beyond fourth-generation mobile radio systems. 